TRANSACTIONS OF THE

AMERICAN MATHEMATICAL SOCIETY

Volume 331, Number 2, June 1992

\title{
PARAMETRIZATION OF A SINGULAR LAGRANGIAN VARIETY
}

\author{
GOO ISHIKAWA \\ Dedicated to Professor Hirosi Toda on his 60th birthday
}

\begin{abstract}
We give stabilization and parametrization theorems for a class of singular varieties in the space of polynomials of one variable and generalize the results of Arnol'd and Givental'. The class contains the open swallowtails and the open Whitney umbrella. The parametrization is associated with the singularity of a stable mapping (in the sense of Thom and Mather) of kernel rank one.
\end{abstract}

\section{INTRODUCTION}

Lagrangian manifold with singularities (singular Lagrangian varieties) arise in many situations. For instance, they arise in the obstacle problem [3], in thermodynamics, in the theory of diffraction [13] or in the study of geodesics of a Riemannian manifold with boundary [2]. They also arise as characteristic varieties of holonomic systems [11] related to the Gauss-Manin connections [17].

We also remark that singular Lagrangian varieties naturally appear in the Cauchy problem of first order partial differential equations: We encounter the situation that the Hamiltonian flow through an isotropic manifold, which corresponds to the initial condition, in a hypersurface $S$ of $T^{*} \mathbf{R}^{n}$ generates a (local) Lagrangian variety, which corresponds to a solution, in $S$.

In many situations stated above, singular Lagrangian varieties are generated in the following reduction process $(*)$ : the set of characteristics through an isotropic manifold $I^{n-1}$ in a hypersurface of a symplectic manifold $M^{2 n}$ is a Lagrangian variety $L$ in the reduced symplectic manifold $N^{2 n-2}$ at least locally.

If the characteristics are not tangent to $I$, then $L$ is an immersed Lagrangian submanifold of $N$. Otherwise, $L$ has singular points parametrized by isotropic mappings of kernel rank one, describing the tangency of characteristics with $I$.

The purpose of this paper is to construct and to analyze some algebraic models of such singular Lagrangian varieties, in the polynomial spaces of one variable.

Related to the variational problem, for instance, the problem of bypassing obstacles, the theory of symplectic triads is developed by Arnol'd and Givental' [4], and an important object "open swallowtail" is obtained.

Received by the editors October 5, 1987 and, in revised form, March 14, 1990.

1980 Mathematics Subject Classification (1985 Revision). Primary 58C27, 58F05, 58C25.

Key words and phrases. Lagrangian variety, isotropic map, parametrization, stratification. 
Open swallowtails are generic singularities of a singular Lagrangian variety associated to a Lagrangian manifold simply tangent to a hypersurface along an isotropic submanifold $I^{n-1}$. (See [1, (5.3.23) and 18, Lecture 3], for the nonsingular case.) As in [3, 6, 12], an open swallowtail is constructed in a polynomial space of one variable and it is described as a "stable object" in some sense.

On the other hand, Givental' [7] shows that, in the problem of Lagrangian immersions of surfaces into $\mathbf{R}^{4}=T^{*} \mathbf{R}^{2}$, the singularity "open Whitney umbrella" defined by $(x, t) \rightarrow\left(q_{1}, q_{2}, p_{2}, p_{1}\right)=\left(x, t^{2} / 2,-t^{3} / 3, x t\right)$ appears stably. He also remark that the open Whitney umbrella is parametrized by a minimal system of generators of the $\mathbf{R}$-algebra,

$$
\{h \in \mathbf{R}[x, t] \mid(\partial h / \partial t)(0)=0\} .
$$

Remark that this algebra is equal to the set of polynomials $h(x, t)$ such that the exterior differential $d h$ is of type $a(x, t) d x+b(x, t) d\left(t^{2} / 2\right)+c(x, t) d(x t)$ for some $a, b, c \in \mathbf{R}[x, t]$.

An analytic version of this was studied already, that is, the relative de Rham cohomology [15]. Let $g: \mathbf{R}^{n}, 0 \rightarrow \mathbf{R}^{p}, 0$ be a map-germ. Consider the ideal $\mathscr{I}$ generated by $d g_{1}, \ldots, d g_{p}$ in the de Rham complex $\Omega$ of germs of differential forms on $\mathbf{R}^{n}, 0$. Then the relative de Rham cohomology $\mathscr{H}_{g}^{i}$ is defined as the $i$ th cohomology of the complex $(\Omega / \mathscr{I}, d)$, where $d: \Omega / \mathscr{I} \rightarrow \Omega / \mathscr{I}$ is the induced homomorphism form the exterior differential of forms. It is interesting to study the structure of $\mathscr{H}_{g}^{i}$ in the singularity theory of mappings, and many results are known in the case $n>p$ (cf. [15]).

Contrary, in the case $n \leq p$, as pointed out in [15], even the structure of the 0th part

$$
\mathscr{H}_{g}^{0}=\left\{h \in \mathscr{O}_{\mathbf{R}^{n}, 0} \mid d h \in\left\langle d g_{1}, \ldots, d g_{p}\right\rangle \mathscr{O}_{\mathbf{R}^{n}, 0}\right\}
$$

is not known in detail.

In this paper, from the motivations stated above, we remark that an open swallowtail and an open Whitney umbrella can be constructed inclusively, by the process $(*)$, in a polynomial space of one variable (Theorem 1 and Proposition $1)$, and we study certain relations between these objects and 0th relative de Rham cohomologies of stable mappings.

We treat an isotropic submanifold $I^{n-1}$ which is not necessarily an intersection of a Lagrangian submanifold and a hypersurface (cf. Remark 5). As a consequence, we have a multidimensional analogue, which is denoted by $W_{2 n}(4 n+1)$ in this paper, of the open Whitney umbrella.

Explicit parametrizations of open swallowtails are given in [6, §3]. These parametrizations relate to the paper [9], where we associate certain ring sheaves with singularities of mappings and discuss the finite generatedness of associated ring sheaves. In the case treated in this paper, these ring sheaves coincide with the sheaves of 0 th relative de Rham cohomologies (see Remark 3).

Derived from this observation, in this paper, more general objects are parametrized (Theorem 2) in a similar way as in [6].

Above all, we emphasize that, for a certain stable mapping (in the sense of Thom and Mather), a minimal system of generators of associated ring sheaf in the sense of [9] turns out a parametrization of certain singular Lagrangian variety (Theorems 3 and 2). 
We can observe that this relation is very natural, since the 0th relative de Rham cohomology $\mathscr{H}_{g}^{0}$ is just the set of generating functions of isotropic liftings $\mathbf{R}^{n}, 0 \rightarrow T^{*} \mathbf{R}^{p}$ of $g$, and it is a deformation space of an isotropic lifting of $g$.

This aspect will be investigated in an another paper.

We also remark that there are important papers, for instance, [10], treating singular Lagrangian varieties on a different viewpoint from this paper. 1.

In $\S 1$, after some preliminaries, we state Theorems 1, 2 and 3 and Proposition

We prove Theorem 3 in $\S 2$, and prove Theorems 1 and 2 in $\S 3$.

As a corollary of Theorem 2 , we give a regular stratification of singular varieties treated in this paper, in $\S 4$.

In the last section, we recall symplectic geometry in polynomial spaces of one variable and prove Proposition 1.

The author would like to thank Professor S. Izumi for valuable comments and thank the referee for helpful advise.

\section{Statements of Results}

Let $\mathbf{K}$ denote $\mathbf{R}$ or $\mathbf{C}$. Set

$$
\mathscr{H}_{n}=\left\{F(t)=\sum_{0 \leq i \leq n} a_{i} t^{n-i} /(n-i) ! \mid a_{i} \in \mathbf{K}\right\},
$$

the K-vector space of polynomials of one variable $t$ with degree $\leq n$. Furthermore set

$$
\mathscr{M}_{n}=\left\{F \in \mathscr{H}_{n} \mid a_{0}=1\right\} \quad \text { and } \quad \mathscr{V}_{n}=\left\{F \in \mathscr{M}_{n} \mid a_{1}=0\right\} .
$$

Define a linear map $D^{-l}: \mathscr{H}_{n} \rightarrow \mathscr{H}_{n+l}(-n \leq l)$ by

$$
D^{-l}\left(t^{j} / j !\right)= \begin{cases}t^{j+l} /(j+l) !, & (0 \leq j+l), \\ 0, & \text { (otherwise) },\end{cases}
$$

and the linearity. $\left(D^{-(-1)}=D\right.$ is just the differentiation by $t$. $)$

Define a polynomial map $\pi: \mathscr{M}_{n} \rightarrow \mathscr{V}_{n-1}$ by

$$
\pi(F(t))=D\left(F\left(t-a_{1}(F)\right)\right)=(D F)\left(t-a_{1}(F)\right) .
$$

For fixed positive integers $n, k$ and $m$, consider the affine subspace $I=$ $I_{n, k}(m)$ of $\mathscr{M}_{m+1},(m \geq(2 k-1) n)$, defined by

in $\mathscr{H}_{m+1}$.

$$
I_{n, k}(m)=D^{-(m+1-n)} \mathscr{M}_{n} \oplus\left(\bigoplus_{1 \leq j \leq k-1} D^{-(m-(2 j+1) n)} \mathscr{H}_{n-1}\right),
$$

Theorem 1. The sequence induced from differentiations by $t$,

$$
\cdots \rightarrow \pi\left(I_{n, k}(m+1)\right) \stackrel{D}{\rightarrow} \pi\left(I_{n, k}(m)\right) \stackrel{D}{\rightarrow} \pi\left(I_{n, k}(m-1)\right) \rightarrow \cdots,
$$

is stabilized at stage $m \geq 2 k n+1$. Precisely, there exists a polynomial section $s: \mathscr{V}_{m} \rightarrow \mathscr{V}_{m+1},(m \geq 2 k n+1)$, of $D=d / d t: \mathscr{V}_{m+1} \rightarrow \mathscr{V}_{m}$, mapping $\pi\left(I_{n, k}(m)\right)$ to $\pi\left(I_{n, k}(m+1)\right)$.

Remark 1. In the case $\mathbf{K}=\mathbf{C}, \pi\left(I_{n, k}(m)\right)$ is an algebraic variety, and $D$ is an isomorphism of algebraic varieties if $m \geq 2 k n+1$. In the case $\mathbf{K}=\mathbf{R}$, $\pi\left(I_{n, k}(m)\right)$ is a semialgebraic set. 
Especially, we are interested in the case $k=1$ and $k=2$. Denote the variety $\pi\left(I_{n, 1}(m)\right)$, (resp. $\left.\pi\left(I_{n, 2}(m)\right)\right) \subset \mathscr{V}_{m}$ by $S_{n}(m)$, (resp. $\left.W_{2 n}(m)\right)$.

Let $A$ be an $n$-dimensional stratified subset of a $2 n$-dimensional complex (resp. real) symplectic manifold $(M, \omega)$. We call $A$ a Lagrangian variety if any holomorphic (resp. $C^{\infty}$ ) mapping $h: N \rightarrow M$ with $h(N) \subset A$ is an isotropic map, that is, $h^{*} \omega=0$.

Proposition 1. The "stable objects" $S_{n}(2 n+1) \subset \mathscr{V}_{2 n+1}$ and $W_{2 n}(4 n+1) \subset \mathscr{V}_{4 n+1}$ are Lagrangian varieties with respect to the natural symplectic structure of $\mathscr{V}_{m}$ for odd $m$. (See $\S 5$.)

The result in Theorem 1 for $S_{n}(m)$ is due to Arnol'd and Givental' [6, 4]. The variety $S_{n}(2 n+1)$ is called the $n$-dimensional open swallowtail. The variety $W_{2}(5)$ is isomorphic to the open Whitney umbrella by a symplectic diffeomorphism.

We seek a concrete parametrization of $\pi\left(I_{n, k}(m)\right)$.

For a polynomial $F(t)$, set

$$
\begin{aligned}
F_{(i)}(t) & =(-1)^{i+1} D^{-1}\left(\left(t^{i} / i !\right) F(t)\right), \\
& =(-1)^{i+1} \int_{0}^{t}\left(u^{i} / i !\right) F(u) d u, \quad(i=0,1,2, \ldots) .
\end{aligned}
$$

For $m \geq(2 k-1) n$, define $Q_{n, k, m}: \mathscr{V}_{n} \times\left(\prod_{k-1} \mathscr{H}_{n-1}\right) \times \mathbf{K} \rightarrow \mathscr{V}_{m}$ by

$$
\begin{gathered}
a_{i}\left(Q_{n, 1, m}(F, y)\right)= \begin{cases}a_{i}(F), & 2 \leq i \leq n, \\
F_{(i-n-1)}(y), & n+1 \leq 1 \leq m,\end{cases} \\
a_{i}\left(Q_{n, k, m}\left(F, G_{1}, \cdots, G_{k-1}, y\right)\right), \quad(k \geq 2), \\
= \begin{cases}a_{i}(F), \quad 2 \leq i \leq n, \\
F_{(i-n-1)}(y), \quad n+1 \leq i \leq 2 n+1, \\
a_{i-2 j n-2}, \quad 2 j n+2 \leq i \leq(2 j+1) n+1, \quad 1 \leq j \leq k \\
G_{j(i-(2 j+1) n-2)}(y), & (2 j+1) n+2 \leq i \leq(2 j+2) n \\
G_{k-1(i-(2 k-1) n-2)}(y), & (2 k-1) n+2 \leq i \leq m,\end{cases}
\end{gathered}
$$

where $F \in \mathscr{V}_{n}$, and $G_{i} \in \mathscr{H}_{n-1},(i=1, \ldots, k-1)$.

Then we have

Theorem 2. Let $\psi: I_{n, k}(m) \rightarrow \mathscr{V}_{m},(m \geq(2 k-1) n)$, denote the restriction of $\pi: \mathscr{M}_{m+1} \rightarrow \mathscr{V}_{m}$.

(1) If $k=1$, then there exists a diffeomorphism $\sigma: I_{n, 1}(m) \rightarrow \mathscr{V}_{n} \times \mathbf{K}$ such that $\psi=Q_{n, 1, m} \circ \sigma$.

(2) If $k \geq 2$, then there exists diffeomorphisms $\sigma: I_{n, k}(m) \rightarrow \mathscr{V}_{n} \times\left(\prod_{k-1} \mathscr{H}_{n-1}\right)$ $\times \mathbf{K}$ and $\tau: \mathscr{V}_{m} \rightarrow \mathscr{V}_{m}$ such that $\psi=\tau \circ Q_{n, k, m} \circ \sigma$.

Remark 2. Let $k=1$ or 2 , and $m=2 k n+1$. Then $\psi$ and $Q$ are isotropic maps. Furthermore if $k=2$, then we can choose $\tau$ a symplectic diffeomorphism at least locally.

The variety $\pi\left(I_{n, k}(2 k n+1)\right)$ is closely related to the stable mapping $f=$ $f_{n, k}: \mathscr{V}_{n} \times\left(\prod_{k-1} \mathscr{H}_{n-1}\right) \times \mathbf{K} \rightarrow \mathscr{V}_{n} \times\left(\prod_{k-1} \mathscr{H}_{n-1}\right) \times \mathbf{K}^{k}$ defined by

$$
\begin{aligned}
& f\left(F, G_{1}, \ldots, G_{k-1}, t\right) \\
& \quad=\left(F, G_{1}, \ldots, G_{k-1}, F_{(0)}(t), G_{1(0)}(t), \ldots, G_{k-1(0)}(t)\right),
\end{aligned}
$$

where $F \in \mathscr{V}_{n}$ and $G_{i} \in \mathscr{H}_{n-1},(i=1, \ldots, k-1)$. 
The mapping $f_{n, k}$ is a stable unfolding of the mapping $\mathbf{K} \rightarrow \mathbf{K}^{k}$ defined by $t \rightarrow\left(-t^{n+1} /(n+1) !, 0, \ldots, 0\right.$ ( $k-1$ times $\left.)\right)$, [8].

The mapping $Q$ (resp. $f)$ can be extended naturally to $\widetilde{Q}: \mathscr{M}_{n} \times\left(\prod_{k-1} \mathscr{H}_{n-1}\right)$ $\times \mathbf{K} \rightarrow \mathscr{M}_{m}$, (resp. $\left.\tilde{f}: \mathscr{M}_{n} \times\left(\prod_{k-1} \mathscr{H}_{n-1}\right) \times \mathbf{K} \rightarrow \mathscr{M}_{n} \times\left(\prod_{k-1} \mathscr{H}_{n-1}\right) \times \mathbf{K}^{k}\right)$.

In general, let $f: M \rightarrow N$ be a mapping. If $\mathbf{K}=\mathbf{C}$ (resp. $\mathbf{K}=\mathbf{R})$, then $f$ is assumed to be polynomial or holomorphic (resp. polynomial, $C^{\omega}$ or $C^{\infty}$ ), according to the situation.

Associated to $f$, define ring sheaves $\mathscr{D}_{f}$ and $\mathscr{H}_{f}^{0}$ by

$$
\begin{aligned}
& \mathscr{D}_{f}(U)=\left\{h \in \mathscr{O}_{M}(U) \mid h_{x}-h(x) \in f^{*} \mathfrak{m}_{f(x)} \cdot \mathscr{O}_{M, x}, \quad(x \in U)\right\}, \\
& \mathscr{H}_{f}^{0}(U)=\left\{h \in \mathscr{O}_{M}(U) \mid d h_{x} \in d\left(f^{*} \mathfrak{m}_{f(x)}\right) \cdot \mathscr{O}_{M, x}, \quad(x \in U)\right\},
\end{aligned}
$$

where $U \subset M$ is an open subset, $\mathscr{O}_{M}$ is the structure sheaf of $M$ in each case, $\mathscr{O}_{M, x}$ is the stalk of $\mathscr{O}_{M}$ at $x, f^{*}: \mathscr{O}_{N, f(x)} \rightarrow \mathscr{O}_{M, x}$ is the induced local homomorphism by $f, \mathfrak{m}_{f(x)} \subset \mathscr{O}_{N, f(x)}$ is the unique maximal ideal and $h_{x}$ means the germ of $h$ at $x$.

Then we have

Theorem 3. The ring sheaf $\mathscr{D}_{\hat{f}_{n, k}}$ (resp. $\left.\mathscr{D}_{f_{n, k}}\right)$ is generated by all components of $\widetilde{Q}_{n, k, 2 k n+1}$ (resp. $Q_{n, k, 2 k n+1}$ ), that is, $\mathscr{D}_{\tilde{f}_{n, k}}=\widetilde{Q}_{n, k, 2 k n+1}{ }^{*} \mathscr{O}_{\mathbf{K}^{2 k n+1}}$, (resp. $\left.\mathscr{D}_{f_{n, k}}=Q_{n, k, 2 k n+1}{ }^{*} \mathscr{O}_{\mathbf{K} 2 k n}\right)$.

Furthermore these form a minimal system of generators of $\mathscr{D}_{\tilde{f}_{n, k}}\left(\right.$ resp. $\left.\mathscr{D}_{f_{n, k}}\right)$ at the origin.

Remark 3. We see $\mathscr{D}_{\tilde{f}_{n, k}}\left(\right.$ resp. $\left.\mathscr{D}_{f_{n, k}}\right)$ coincides with $\mathscr{H}_{\hat{f}_{n, k}}^{0}\left(\right.$ resp. $\left.\mathscr{H}_{f_{n, k}^{0}}^{0}\right)$. (See $\S 2$, proof of Theorem 3.)

\section{MiNiMAL SYSTEM OF GENERATORS}

We use notations in $\S 1$.

For the proof of Theorems 1, 2 and 3, we use the following

Lemma 1. There exist $A_{m, i} \in \mathbf{Q}\left[x_{0}, x_{1}, \ldots, x_{n}\right],(0 \leq i \leq n, m=0,1,2$, $\ldots)$, such that, for any $F=t^{n} / n !+\sum_{1 \leq i \leq n} a_{i} t^{n-i} /(n-i) ! \in \mathscr{M}_{n}$,

$$
F_{(m)}=\sum_{1 \leq i \leq n} A_{m, i}\left(F_{(0)}, a_{1}, \ldots, a_{n}\right) F_{(i)}+A_{m, 0}\left(F_{(0)}, a_{1}, \ldots, a_{n}\right),
$$

$(m=0,1,2, \ldots)$, and that $\operatorname{ord}_{0} A_{m, i} \rightarrow \infty$ as $m \rightarrow \infty$.

Proof. There are rational numbers $r_{i},(0 \leq i \leq n)$, such that, for any $F \in \mathscr{M}_{n}$,

$$
F_{(n+m+1)}=r_{0} F_{(0)} F_{(m)}+\sum_{1 \leq i \leq n} r_{i} a_{i} F_{(n+m+1-i)} \quad(m=0,1,2, \ldots) .
$$

In fact, it is sufficient to set

$$
r_{0}=1 /\left\{\left(\begin{array}{c}
n+m \\
m
\end{array}\right)+\left(\begin{array}{c}
n+m+1 \\
m
\end{array}\right)\right\}
$$

and

$$
r_{i}=-r_{0}\left\{\left(\begin{array}{c}
n+m-i \\
m
\end{array}\right)+\left(\begin{array}{c}
n+m+1-i \\
m
\end{array}\right)\right\}, \quad(1 \leq i \leq n) .
$$


Thus $F_{(m)},(m \geq n+1)$, is a linear homogeneous function of $F_{(m-1)}, \ldots$, $F_{(m-n-1)}$ with coefficients in $\mathbf{Q}\left[F_{(0)}, a_{1}, \ldots, a_{n}\right]$ with order $\geq 1$ at the origin. Using this relation iteratively, we have required $A_{m, i}$.

We need the following lemma for the proof of Theorem 3 in the holomorphic and $C^{\omega}$ cases.

Lemma 2. Let $h:\left(\mathbf{K}^{n}, 0\right) \rightarrow\left(\mathbf{K}^{p}, 0\right)$ be an analytic and finite map-germ and $\hat{g} \in F_{0}=\mathbf{K}\left[\left[y_{1}, \ldots, y_{p}\right]\right]$ be a formal power series. If $\hat{g} \circ h$ is analytic, then there exists an analytic $g \in \mathscr{O}_{0}=\mathbf{K}\left\{\left\{y_{1}, \ldots, y_{p}\right\}\right\}$ such that $g \circ h=\hat{g} \circ h$.

Proof. In general, let $\varphi:(A, \mathfrak{m}) \rightarrow(B, \mathfrak{n})$ be a local and unitary homomorphism of local rings. Assume $B$ is a finite $A$-module via $\varphi$. Then $\operatorname{Im} \varphi$ is closed with respect to $\mathfrak{n}$-adic topology. (This is kindly communicated to the author by S. Izumi.)

In fact, by Artin-Rees lemma, $\operatorname{Im} \varphi$ is closed with respect to m-adic topology $\left[14,3.13\right.$ (iii)]. Since $\mathfrak{n}^{k} B \subset \mathfrak{m} B \subset \mathfrak{n} B$ for some $k \in \mathbf{N}$, m-adic topology of $B$ concides with $\mathfrak{n}$-adic one.

The closure of $\operatorname{Im} \varphi$ is equal to $\operatorname{Im} \hat{\varphi} \cap B$, where $\hat{\varphi}: \widehat{A} \rightarrow \widehat{B}$ is the completion of $\varphi$. Thus we see $\operatorname{Im} \varphi=\operatorname{Im} \hat{\varphi} \cap B$.

Applying this to $\varphi=h^{*}: \mathscr{O}_{\mathbf{K}^{p}, 0} \rightarrow \mathscr{O}_{\mathbf{K}^{n}, 0}$, we have Lemma 2.

Proof of Theorem 3 (first half). The first half of Theorem 3 for the original $Q$ and $f$ follows easily from that for the extended $\widetilde{Q}$ and $\tilde{f}$.

We also treat complex and real formal cases. Then the structure sheaf of $\mathbf{K}^{p}$ is defined by $\mathscr{O}(U)=\prod_{x \in U} \mathscr{F}_{x}$, where $\mathscr{F}_{x}=\mathscr{E}_{x} /\left(\bigcap_{1<i} \mathfrak{m}^{i}\right) \cong \mathbf{K}\left[\left[y_{1}, \ldots, y_{p}\right]\right]$, $\mathscr{E}_{x}$ is the ring of germs of $C^{\infty}$-functions at $x$ and $U \subset \mathbf{K}^{p}$ is an open subset.

Set $X=\mathscr{M}_{n} \times\left(\prod_{k-1} \mathscr{H}_{n-1}\right) \times \mathbf{K} \cong \mathbf{K}^{k n+1}$.

Let $\Lambda_{u},(u=0,1,2, \ldots)$, denote the set of $\left(F, G_{1}, \ldots, G_{k-1}, t\right) \in X$ satisfying

$$
\left(D^{j} F\right)(t)=\left(D^{j} G_{1}\right)(t)=\cdots=\left(D^{j} G_{k-1}\right)(t)=0, \quad(0 \leq j \leq u-1) .
$$

Define an ideal sheaf $\mathscr{F}_{\tilde{f}}$ by

$$
\mathscr{I}_{\hat{f}}(U)=\left\{k \in \mathscr{O}(U) \mid D^{u} k \text { vanishes on } \Lambda_{u+1},(u=0,1,2, \ldots)\right\} \text {, }
$$

where $U \subset X$ is an open subset.

Then, by the proof of Proposition 3.1 of [9], we see

$$
\mathscr{D}_{\hat{f}}(U)=\left\{k \in \mathscr{O}(U) \mid D k \in \mathscr{F}_{\hat{f}}(U)\right\}=\mathscr{H}_{\hat{f}}^{0}(U) .
$$

(This is valid in all cases which we are treating.)

We remark that $\mathscr{F}_{\hat{f}}$ is generated by $F, G_{1}, \ldots, G_{k-1}$, considered as polynomial elements of $\mathscr{O}(X)$. In fact, since $F, G_{1}, \ldots, G_{k-1}$ can be taken as a part of coordinates of $X$, the set of sections over $U$ of the ideal sheaf generated by $F, G_{1}, \ldots, G_{k-1}$ is equal to $\left\{k \in \mathscr{O}(U) \mid k\right.$ vanishes on $\left.\Lambda_{1}\right\}$, which contains $\mathscr{\mathscr { F }}_{\hat{f}}(U)$.

In the polynomial case, for any $k \in \mathscr{D}_{\hat{f}, x}$,

$$
D k=A_{0} F+A_{1}\left(F+G_{1}\right)+\cdots+A_{k-1}\left(F+G_{k-1}\right),
$$

for some polynomials $A_{i} \in \mathscr{O}_{x}$. Then,

$$
k=k(x)+D^{-1}\left(A_{0} F\right)+\sum_{1 \leq i \leq k-1} D^{-1}\left(A_{i}\left(F+G_{i}\right)\right),
$$


using the notation in $\S 1$. By Lemma 1 , each term in the right-hand side is a polynomial of components of $\widetilde{Q}_{n, k, 2 k n+1}$ and $G_{i(n)} \quad(1 \leq i \leq k-1)$.

Furthermore, setting $R(t)=F(t)-t^{n} / n !$,

$$
\begin{aligned}
G_{i(n)} & =(-1)^{n+1} \int_{0}^{t}\left(u^{n} / n !\right) G_{i}(u) d u \\
& =(-1)^{n+1} \int_{0}^{t}\left\{F(u) G_{i}(u)-R(u) G_{i}(u)\right\} d u .
\end{aligned}
$$

Therefore $G_{i(n)}$ is the linear combination of $F_{(0)}, \ldots, F_{(n)}, G_{i(0)}, \ldots$, $G_{i(n-1)}$. Thus we have required result in the polynomial case.

For the $C^{\infty}$, complex formal and real formal cases, we follow the proof of Proposition 2.2 of [9]. The different point is only the usage of Lemma 1 instead of Lemma 2.6 of [9].

By the finiteness of $\widetilde{Q}$ and by Lemma 2, the result in the holomorphic (resp. $C^{\omega}$ ) case follows from that in the complex (resp. real) formal case.

Proof of Theorem 3 (Second half). Set $\widetilde{Q}=\widetilde{Q}_{n, k, 2 k n+1}$. Let

$$
\tau=\tau\left(a_{i}, b_{j i}, c_{l}, d_{j m} \mid 1 \leq i \leq n, 1 \leq j \leq k-1,0 \leq l \leq n, 0 \leq m \leq n-1\right)
$$

be a formal power series with coefficients in $\mathbf{K}$. Assume $\tau \circ \widetilde{Q}=0$. Then it is sufficient to show $\operatorname{ord}_{0} \tau \geq 2$.

First, restricting the relation $\tau \circ \widetilde{Q}=0$ to the subspace $\{t=0\}$, we see $\partial \tau / \partial a_{i}(0)=\partial \tau / \partial b_{j i}(0)=0,(1 \leq i \leq n, 1 \leq j \leq k-1)$.

Second, for fixed $j_{0},\left(1 \leq j_{0} \leq k-1\right)$, restrict $\tau \circ \widetilde{Q}=0$ to the subspace $\left\{a=0, b_{j i}=0\right.$ if $\left.(\bar{j}, i) \neq\left(j_{0}, n\right)\right\}$. Since $b_{j_{0}, n}, t^{n+1}, \ldots, t^{2 n+1}$, $b_{j_{0}, n} t, \ldots, b_{j_{0}, n} t^{n}$ has no formal relation of order $\leq 1$, we see $\partial \tau / \partial c_{l}(0)=$ $\partial \tau / \partial d_{j_{0}, m}(0)=0,(0 \leq l \leq n, 0 \leq m \leq n-1)$.

Thus $\operatorname{ord}_{0} \tau \geq 2$. Similarly we have that $\tau \circ \widetilde{Q}=0$ implies $\operatorname{ord}_{0} \tau \geq 2$. This completes the proof of Theorem 3 .

\section{Stabilization and PaRAMETRIZATION}

For $F \in \mathscr{H}_{m}$ and $y \in \mathbf{K}$, set $\Phi_{y}(F(t))=F(t+y)$. Then $\Phi_{y}: \mathscr{H}_{m} \rightarrow \mathscr{H}_{m}$ is a linear automorphism preserving $\mathscr{M}_{m}$.

We need the following lemma, which is an easy consequence of integration by part:

Lemma 3. Let $F \in \mathscr{H}_{n}$, and $l>0$. Then

$$
\Phi_{-y} \circ D^{-l-1} \circ \Phi_{y} F=D^{-l-1} F-\int_{0}^{y}\left\{(t-u)^{l} / l !\right\} F(u) d u,
$$

for any $y \in \mathbf{K}$.

Define $\Phi: \mathscr{M}_{m} \times \mathbf{K} \rightarrow \mathscr{M}_{m}$ by $\Phi(F, y)=\Phi_{-y} F$. Then, $\Phi^{-1}\left(\mathscr{V}_{m}\right)=\{(F, y) \in$ $\left.\mathscr{M}_{m} \times \mathbf{K} \mid y=a_{1}(F)\right\}$. Set $i(F)=\left(F, a_{1}(F)\right) \in \mathscr{M}_{m} \times \mathbf{K}$. Then $\pi: \mathscr{M}_{m+1} \rightarrow \mathscr{V}_{m}$ is factorized as $\Phi \circ i \circ D$.

Notice that $D I_{n, k}(m)=I_{n, k}(m-1)$. Let $\varphi: I_{n, k}(m-1) \times \mathbf{K} \rightarrow \mathscr{M}_{m}$ be the restriction of $\Phi$. (This is a similar construction as [1, (5.3.29)].)

Proof of Theorems 1 and $2(k=1)$. First we will parametrize $\varphi$. Define $\Sigma: I_{n, 1}(m-1) \times \mathbf{K} \rightarrow \mathscr{M}_{n} \times \mathbf{K}$ by $\Sigma(G, y)=\left(\Phi_{-y} D^{m-n} G, y\right)$. Then $\Sigma$ is 
a polynomial diffeomorphism. Define $Q_{m}^{\prime}: \mathscr{M}_{n} \times \mathbf{K} \rightarrow \mathscr{M}_{m}$ by $Q_{m}^{\prime}(F, y)=$ $\Phi_{-y} \circ D^{-(m-n)} \circ \Phi_{y}(F)$. Then we have $(\mathrm{a}): \varphi=Q_{m}^{\prime} \circ \Sigma$.

Thus $\psi=\pi\left|I_{n, 1}(m)=Q_{m}^{\prime} \circ \Sigma \circ i \circ D\right| I_{n, 1}(m)$. Set $\sigma=\Sigma \circ i \circ D \mid I_{n, 1}(m)$ : $I_{n, 1}(m) \rightarrow \mathscr{V}_{n} \times \mathbf{K}$. Then $\sigma$ is a polynomial diffeomorphism.

Then we have (b): $Q_{n, 1, m}=Q_{m}^{\prime} \mid \mathscr{\mathscr { V }}_{n} \times \mathbf{K}$.

In fact, by Lemma 3 , for $(F, y) \in \mathscr{M}_{n} \times \mathbf{K}$,

$$
\begin{aligned}
Q_{m}^{\prime}(F, y) & =D^{-(m-n)} F-\int_{0}^{y}\left\{(t-u)^{m-n-1} /(m-n-1) !\right\} F(u) d u \\
& =D^{-(m-n)} F+\sum_{0 \leq i \leq m-n-1} F_{(i)}(y) t^{m-n-1-i} /(m-n-1-i) ! .
\end{aligned}
$$

This shows Theorem 2(1).

Next we will show that (c): for $2 n+1 \leq l \leq m$, there exists a polynomial map $s_{m, l}: \mathscr{M}_{l} \rightarrow \mathscr{M}_{m}$ such that $Q_{m}^{\prime}=s_{m, l} \circ Q_{l}^{\prime}$ and that $D^{m-l} \circ s_{m, l}=\operatorname{id}_{\mathscr{M}_{l}}$.

By Lemma 1 or Theorem 3, for $l<j \leq m$, there exists a polynomial $A_{j} \in$ $\mathbf{K}\left[a_{1}, \ldots, a_{n} ; x_{0}, \ldots, x_{n}\right]$ such that

$$
F_{(j-n-1)}(y)=A_{j}\left(a_{1}(F), \ldots, a_{n}(F) ; F_{(0)}(y), \ldots, F_{(n)}(y)\right) .
$$

Then, to see (c), it is sufficient to define $s_{m, l}$ by

$$
a_{i}\left(s_{m, l}(G)\right)=\left\{\begin{array}{l}
a_{i}(G), \quad 1 \leq i \leq l, \\
A_{i}\left(a_{1}(G), \ldots, a_{2 n+1}(G)\right), \quad l+1 \leq i \leq m .
\end{array}\right.
$$

Set $s=s_{m+1, m} \mid \mathscr{V}_{m}: \mathscr{V}_{m} \rightarrow \mathscr{V}_{m+1}$. Then $s$ is a polynomial section of $D$, and $s\left(\pi\left(I_{n, 1}(m)\right)\right)=s \circ Q_{m}^{\prime}\left(\mathscr{V}_{n} \times \mathbf{K}\right)=Q_{m+1}^{\prime}\left(\mathscr{V}_{n} \times \mathbf{K}\right)=\pi\left(I_{n, 1}(m+1)\right)$, by (a). This shows Theorem 1, $(k=1)$.

Proof of Theorems 1 and 2 for general $k$. Define $Q_{k, m}^{\prime}: \mathscr{M}_{n} \times\left(\prod_{k-1} \mathscr{H}_{n-1}\right) \times$ $\mathbf{K} \rightarrow \mathscr{M}_{m}$ by

$$
\begin{aligned}
& Q_{k, m}^{\prime}\left(F, G_{1}, \ldots, G_{k-1}, y\right) \\
& \quad=\Phi_{-y} \circ D^{-(m-n)} \circ \Phi_{y} F+\sum_{1 \leq j \leq k-1} \Phi_{-y} \circ D^{-(m-(2 j+1) n)} \circ \Phi_{y} G_{j} .
\end{aligned}
$$

There is a natural isomorphism $\lambda: \mathscr{M}_{n} \times\left(\prod_{k-1} \mathscr{H}_{n-1}\right) \rightarrow I_{n, k}(m-1)$, that is,

$$
\lambda\left(F, G_{1}, \ldots, G_{k-1}\right)=D^{-(m-n)} F+\sum_{1 \leq j \leq k-1} D^{-(m-(2 j+1) n-1)} G_{j} .
$$

Define $\Sigma: I_{n, k}(m-1) \times \mathbf{K} \rightarrow \mathscr{M}_{n} \times\left(\prod_{k-1} \mathscr{H}_{n-1}\right) \times \mathbf{K}$ by

$$
\Sigma(G, y)=\left(\Phi_{-y} F, \Phi_{-y} G_{1}, \ldots, \Phi_{-y} G_{k-1}, y\right),
$$

where $\lambda^{-1} G=\left(F, G_{1}, \ldots, G_{k-1}\right)$.

Then we see (a): $\varphi=Q_{k, m}^{\prime} \circ \Sigma$.

By Lemma 3, the coefficient $a_{\nu}$ of $t^{m-\nu} /(m-\nu)$ ! of

$$
Q_{k, m}^{\prime}\left(F, G_{1}, \ldots, G_{k-1}, y\right) \in \mathscr{M}_{m}
$$

is equal to

$$
F_{(\nu-n-1)}(y)+\sum_{1 \leq j \leq k-1} G_{j(\nu-(2 j+1) n-1)}(y),
$$


for $2 k n+1 \leq \nu \leq m$. Then by Theorem 3 (polynomial case), there exists $A_{\nu} \in \mathbf{K}\left[\mathscr{M}_{2 k n+1}\right]$ such that $a_{\nu}=A_{\nu} \circ Q_{k, 2 k n+1}^{\prime}$.

We see (c): for $2 k n+1 \leq l \leq m$, there exists a polynomial map $s_{m, l}: \mathscr{M}_{l} \rightarrow$ $\mathscr{M}_{m}$ such that $Q_{k, m}^{\prime}=s_{m, l} \circ Q_{k, l}^{\prime}$ and that $D^{m-l} \circ s_{m, l}=\operatorname{id}_{\mathscr{M}_{l}}$.

In fact, it is sufficient to set, for $G \in \mathscr{M}_{l}$,

$$
a_{i}\left(s_{m, l}(G)\right)=\left\{\begin{array}{l}
a_{i}(G), \quad 1 \leq i \leq l, \\
A_{i}\left(a_{1}(G), \ldots, a_{2 k n+1}(G)\right), \quad l+1 \leq i \leq m .
\end{array}\right.
$$

Setting $s=s_{m+1, m} \mid \mathscr{V}_{m}$, we have Theorem 1 in general.

Next we will show (b): there exists a polynomial diffeomorphism $T: \mathscr{M}_{m} \rightarrow$ $\mathscr{M}_{m}$ preserving $\mathscr{V}_{m}$ such that $Q_{k, m}^{\prime}=T \circ Q_{n, k, m}$.

Set $\xi=Q_{k, m}^{\prime}\left(F, G_{1}, \ldots, G_{k-1}, y\right)$ and $\eta=Q_{n, k, m}\left(F, G_{1}, \ldots, G_{k-1}, y\right)$. Then, by Lemma 3 and Theorem 3 (polynomial case), each coefficient $a_{\nu}(\xi-\eta)$, $(2 n+1<\nu)$, of the difference $\xi-\eta$ is a polynomial of preceding coefficients of $\eta ; a_{\nu}(\xi-\eta)=A_{\nu}\left(a_{1}(\eta), a_{2}(\eta), \ldots, a_{\nu-1}(\eta)\right)$.

Then define $T$ by

$$
T\left(a_{1}, \ldots, a_{m}\right)=\left(a_{1}, \ldots, a_{2 n+1}, a_{2 n+2}+A_{2 n+2}(a), \ldots, a_{m}+A_{m}(a)\right) .
$$

We easily see $T$ satisfies the required property of (b).

By (b), $\psi=T \circ Q_{n, k, m} \circ \Sigma \circ i \circ D \mid I_{n, k}(m)$.

Set $\sigma=\sum \circ i \circ D \mid I_{n, k}(m): I_{n, k}(m) \rightarrow \mathscr{V}_{n} \times\left(\prod_{k-1} \mathscr{H}_{k-1}\right) \times \mathbf{K}$ and $\tau=T \mid \mathscr{V}_{m}$. Then we have $\psi=\tau \circ Q_{n, k, m} \circ \sigma$. Thus Theorem 2(2) is proved.

This completes the proof of Theorems 1 and 2 .

Remark 4. In the above proof, though $Q_{m}^{\prime}$ can be extended naturally to $Q_{m}^{\prime}$ : $\mathscr{H}_{n} \times \mathbf{K} \rightarrow \mathscr{H}_{m}$, the statement (c) does not hold for extended $Q_{m}^{\prime}$.

\section{Stratification}

We deduce from Theorem 2 a property of the stable object $\pi\left(I_{n, k}(2 k n+1)\right)$.

In this section, we identify $\mathscr{V}_{n} \times\left(\prod_{k-1} \mathscr{H}_{n-1}\right) \times \mathbf{K}$ with $\mathbf{K}^{k n}$ by the correspondence $\left(F, G_{1}, \ldots, G_{k-1}, t\right) \rightarrow\left(a(F), a\left(G_{1}\right), \ldots, a\left(G_{k-1}\right), t\right)$.

Lemma 4. Let $m \geq 2 n+1$ if $k=1$, and $m \geq(2 k-1) n+1$ if $k \geq 2$. Then $\psi: I_{n, k}(m) \rightarrow \mathscr{V}_{m}$ is a homeomorphism onto the image.

Proof. By Theorem 2, it is sufficient to show that $Q=Q_{n, k, m}$ is a homeomorphism onto the image.

First $Q$ is continuous and injective. In fact, let $Q\left(F, G_{1}, \ldots, G_{k-1}, t\right)=$ $Q\left(F^{\prime}, G_{1}^{\prime}, \ldots, G_{k-1}^{\prime}, t^{\prime}\right)$. Then $F=F^{\prime}, G_{j}=G_{j}^{\prime}(1 \leq j \leq k-1)$, and $F_{(i)}(t)=F_{(i)}\left(t^{\prime}\right), \quad(0 \leq i \leq n)$. By Lemma 2.7 of [9], we see $t=t^{\prime}$.

Furthermore $Q$ is proper, since already the mapping $\left(F, G_{1}, \ldots, G_{k-1}, t\right)$ $\rightarrow\left(F, G_{1}, \ldots, G_{k-1}, F_{(0)}(t)\right)$ is proper.

Thus we have Lemma 4.

Two stratified sets $(A, \mathscr{A}) \subset \mathbf{K}^{r}$ and $(B, \mathscr{B}) \subset \mathbf{K}^{s}$ are called isomorphic if there is a homeomorphism $\sigma: A \rightarrow B$ mapping diffeomorphically each stratum of $\mathscr{A}$ onto a stratum of $\mathscr{B}$.

Corollary 1. The variety $\pi\left(I_{n, k}(2 k n+1)\right)$ has a Whitney regular stratification $\mathscr{T}$ isomorphic to the stratification of $\mathbf{K}^{k n}$ by the Thom-Boardman stratification of $f_{n, k}: \mathbf{K}^{k n} \rightarrow \mathbf{K}^{k n+k-1}$.

The dimension of each stratum of $\mathscr{T}$ is a multiple of $k$. 
See $[19, \S 19$ and $16, \S 2]$ for the Whitney regularity.

Proof. By Theorem 2, $\pi\left(I_{n, k}(2 k n+1)\right)$ is parametrized by $Q=Q_{n, k, 2 k n+1}$. The decomposition of $\mathbf{K}^{k n}$ by the Thom-Boardman singularities of $Q$ coincides with that of $f=f_{n, k}$;

$$
\mathbf{K}^{k n}=\bigcup_{0 \leq i \leq n} \Sigma_{(i)}(Q)=\bigcup_{0 \leq i \leq n} \Sigma_{(i)}(f),
$$

where $\Sigma_{(i)}=\Sigma^{1,1, \ldots, 1},(i$ times $)$. (See [5].)

Further we have $\Sigma_{(i)}(Q)=\left\{\left(F, G_{1}, \ldots, G_{k-1}, t\right) \in \mathbf{K}^{k n} \mid t\right.$ is a common root of $F, G_{1}, \ldots, G_{k-1}$ of multiplicity $\left.\geq i\right\}$.

We see $\Sigma_{(i)}(Q)$ is a submanifold of codimension $k i$.

By Lemma 4, $Q$ is a homeomorphism onto $\pi\left(I_{n, k}(2 k n+1)\right)$. Therefore $Q \mid \Sigma_{(i)}(Q)$ is a diffeomorphism onto the image, by the definition of ThomBoardman singularity.

Set $\mathscr{T}=\left\{Q\left(\Sigma_{(i)}(Q)\right\}_{0 \leq i \leq n}\right.$.

Let $x \in \Sigma_{(i)}(f)$. Then the germ $f: \mathbf{K}^{k n}, x \rightarrow \mathbf{K}^{k n+k-1}$ is right-left equivalent to the germ of $f_{n-i, k} \times \mathrm{id}_{\mathbf{K}^{k i}}$ at 0 . Thus $\mathscr{D}_{f}$ is isomorphic to $\mathscr{D}_{f_{n-i, k}} \times \mathrm{id}_{\mathbf{K}^{k i}}$. Then we see the germ of $Q$ at $x$ is right-left equivalent to the germ at 0 of $\left(Q_{n-i, k, 2 k(n-i)+1} \times \mathrm{id}_{\mathbf{K}^{k i}}, 0\right): \mathbf{K}^{k n} \rightarrow \mathbf{K}^{2 k n-k i} \times \mathbf{K}^{k i}$, where $0: \mathbf{K}^{k n} \rightarrow \mathbf{K}^{k i}$ is the constant zero map.

Therefore, for each $y \in Q\left(\Sigma_{(i)} Q\right)$, there exists a germ of diffeomorphism $\mathbf{K}^{2 k n}, y \rightarrow \mathbf{K}^{2 k(n-i)} \times \mathbf{K}^{2 k i}, 0$ mapping $\mathscr{T}$ to $\mathscr{T}^{\prime} \times \mathbf{K}^{k i} \times 0$ and $Q\left(\Sigma_{(i)} Q\right)$ to $0 \times \mathbf{K}^{k i} \times 0$, where $\mathscr{T}^{\prime}$ is the stratification associated to $f_{n-i, k}$.

Thus $\mathscr{T}$ is Whitney regular along $Q\left(\Sigma_{(i)} Q\right),(0 \leq i \leq n)$.

\section{LAGRANGIAN PROPERTY}

We recall the symplectic structures on $\mathscr{H}_{2 n+1}, \mathscr{M}_{2 n}$ and $\mathscr{V}_{2 n-1},[4, \S 1]$.

Set $\Phi_{y}(F(t))=F(t+y),\left(y \in \mathbf{K}, F \in \mathscr{H}_{2 n+1}\right)$. Then $\Phi_{y}: \mathscr{H}_{2 n+1} \rightarrow \mathscr{H}_{2 n+1}$ is a linear symplectic transformation with respect to the symplectic structure $\omega=\sum_{0 \leq i \leq n} d p_{i} \wedge d p_{i}$, where $q_{i}=a_{n-i}, p_{i}=(-1)^{i+1} a_{n+i+1},(0 \leq i \leq n)$.

The flow $\left\{\Phi_{y}\right\}_{y \in \mathbf{K}}$ is a Hamiltonian flow with the Hamiltonian

$$
h=\frac{1}{2} \sum_{0 \leq i \leq 2 n}(-1)^{n+i} a_{i} a_{2 n-i}=\frac{q_{0}^{2}}{2}+\sum_{0 \leq i \leq n-1} p_{i} q_{i+1} \text {. }
$$

In fact, $X=\sum_{1 \leq i \leq 2 n+1} a_{i-1} \partial / \partial a_{i}$ is the infinitesimal transformation of $\left\{\Phi_{y}\right\}_{y \in K}$, and $\left.X\right\rfloor \omega=-d h$.

Notice that $h$ is independent of $a_{2 n+1}$.

The field $\partial / \partial a_{2 n+1}$ is a characteristic field on the hypersurface $\left\{a_{0}=1\right\}$. Then the space of characteristics is identified with $\mathscr{M}_{2 n}$ and the induced symplectic structure on $\mathscr{M}_{2 n}$ is $\omega=\sum_{0 \leq i \leq n-1} d p_{i} \wedge d q_{i}$. The induced function

$$
h=q_{0}^{2} / 2+p_{0} q_{1}+\cdots+p_{n-2} q_{n-1}+p_{n-1}
$$

is a Hamiltonian of the induced flow $\left\{\Phi_{y}\right\}_{y \in \mathbf{K}}$ on $\mathscr{M}_{2 n}$.

The space of characteristics of hypersurface $\{h=0\}$ is identified with $\mathscr{V}_{2 n-1}$ by the mapping $\pi:\{h=0\} \subset \mathscr{M}_{2 n} \rightarrow \mathscr{V}_{2 n-1}$, (cf. $\S 1$ ). Then the induced symplectic structure on $\mathscr{V}_{2 n-1}$ is $\omega=\sum_{0 \leq i \leq n-2} d p_{i} \wedge d q_{i}$. 
Lemma 5. If $k=1$ or 2 , then $\psi: I_{n, k}(2 k n+1) \rightarrow \mathscr{V}_{2 k n+1}$ is isotropic, that is, $\psi^{*} \omega=0$.

Proof. If $k=1$ or 2 , then $I_{n, k}(2 k n+1) \subset\{h=0\}$. Furthermore, the symplectic form $\omega_{\mathscr{M}_{2 k n+2}}$ vanishes on $I_{n, k}(2 k n+1)$. Thus

$$
\psi^{*} \omega_{\mathscr{V}_{2 k n+1}}=\omega_{\mathscr{M}_{2 k n+2}} \mid I_{n, k}(2 k n+1)=0 .
$$

Corollary 2. Each stratum of $\left(S_{n}(2 n+1), \mathscr{T}\right),\left(\right.$ resp. $\left.\left(W_{2 n}(4 n+1), \mathscr{T}\right)\right)$ is an isotropic manifold.

Proof of Proposition 1. Let $h: N \rightarrow M$ satisfy $h(N) \subset S_{n}(2 n+1)$ (resp. $\left.W_{2 n}(4 n+1)\right)$. Denote by $U_{i}$ the interior of $h^{-1} Q\left(\Sigma_{(i)} Q\right)$. Then $h^{*} \omega \mid U_{i}=0$, $(0 \leq i \leq n)$, and $N=\bigcup_{0 \leq i \leq n} \bar{U}_{i}$. Thus $h^{*} \omega=0$.

Remark 5. $I_{n, 1}(2 n+1) \subset \mathscr{M}_{2 n+2}$ is an intersection of the Lagrangian manifold $I_{n+1,1}(2 n+1)$ and the hypersurface $\{h=0\}$. On the other hand, $I_{n, 2}(4 n+1) \subset$ $\mathscr{M}_{4 n+2}$ is not an intersection of any Lagrangian manifold and $\{h=0\}$.

Proof. Set $X=I_{n, 2}(2 n+1)$. Suppose $L$ is a Lagrangian submanifold containing $X$. Then there exist disjoint subsets $I$ and $J$ of $\{0,1, \ldots, 2 n\}$ and a smooth function $S=S\left(q_{I}, p_{J}\right)$ such that $I \cup J=\{0,1, \ldots, 2 n\}, J \supset$ $\{0,1, \ldots, n-1\}, I \supset\{n+1, \ldots, 2 n\}$ and $L$ is defined by $p_{I}=\left(\partial S / \partial q_{I}\right)$, $q_{J}=-\left(\partial S / \partial p_{J}\right)$. Then we have two cases: (1) $n \in I,(2) n \notin I$. In the case (1), by simple calculations, we see that $h \mid L=q_{n}\left(p_{n+1}+\right.$ higher term) with respect to the coordinates $q_{I}, p_{J}$ of $L$. Similarly, in the case (2), we have $h \mid L=p_{n}\left(q_{n+1}+\right.$ higher term). In any case, we see that $L \cap\{h=0\}$ is not equal to $X$ as germ at 0 .

\section{REFERENCES}

1. R. Abraham and J. E. Marsden, Foundation of mechanics, 2nd ed., Benjamin, New York, 1978.

2. S. B. Alexander, I. D. Berg, and R. L. Bishop, Cauchy uniqueness in the Riemannian obstacle problem, Lecture Notes in Math., vol. 1209, Springer-Verlag, 1986, pp. 1-7.

3. V. I. Arnol'd, Lagrangian manifolds with singularities, asymptotic rays and the open swallowtail, Funct. Anal. Appl. 15 (1981), 235-246.

4. __ Singularities in variational calculus, Itogi Nauki, Contemporary Problems in Mathematics 22 (1983), 3-55; English transl., J. Soviet Math. 27 (1984), 2679-2713.

5. Th. Bröcker, Differential germs and catastrophes, London Math. Soc. Lecture Notes Series 17, Cambridge Univ. Press, 1975.

6. A. B. Givental', Manifolds of polynomials having a root of fixed multiplicity, and the generalized Newton equation, Funct. Anal. Appl. 16 (1982), 10-14.

7. L__ Lagrangian imbeddings of surfaces and unfolded Whitney umbrella, Funct. Anal. Appl. 20 (1986), 197-203.

8. M. Golubitsky and V. W. Guillemin, Stable mappings and their singularities, SpringerVerlag, 1973.

9. G. Ishikawa, Families of functions dominated by distributions of $\mathscr{C}$-classes of mappings, Ann. Inst. Fourier (Grenoble) 33 (1983), 199-217.

10. S. Janeczko, Generating families for images of Lagrangian submanifolds and open swallowtails, Math. Proc. Cambridge Philos. Soc. 100 (1986), 91-107.

11. ___ Constrained Lagrangian submanifolds over singular constraining varieties and discriminant varieties, Ann. Inst. Henri Poincaré 46 (1987), 1-26. 
12. Tensor invariants and invariant symplectic geometry of binary forms, Bull. Polon. Acad. Sci. Math. 36 (1988), 15-23.

13. J. Keller, A geometrical theory of diffraction, Proc. Sympos. Appl. Math., vol. 8, Amer. Math. Soc., Providence, R.I., 1958, pp. 27-52.

14. B. Malgrange, Ideals of differentiable functions, Oxford Univ. Press, 1966.

15. __, Frobenius avec singularités, 2. Le cas général, Invent. Math. 39 (1977), 67-89.

16. J. N. Mather, Notes on topological stability, Harvard Univ. preprint, 1970.

17. A. N. Varchenko and A. B. Givental', Period mapping and intersection form, Funktsional Anal. i Prilozhen 16 (1982), 7-20=Functional Anal. Appl. 16 (1982), 83-93.

18. A. Weinstein, Lectures on symplectic manifolds, CBMS Regional Conf. Ser. in Math., no. 29, Amer. Math. Soc., Providence, R.I., 1977.

19. H. Whitney, Tangents to an analytic variety, Ann. of Math. (2) 81 (1964), 496-549.

Department of Mathematics, Faculty of Sciences, Hokkaido University, Sapporo 060, JAPAN

E-mail address: f13527@jpnkudpc.bitnet 